\title{
Adherencia al tratamiento de la hipertensión
}

\section{arterial}

\author{
Adherence to the treatment of arterial hypertension
}

\section{Adherencia al tratamiento de la hipertensión arterial}

\author{
María Pincay Cañarte. Mg.
}

Universidad Estatal del Sur de Manabí, maria.pincay@unesum.edu.ec, orcid.org/0000-0003-4110-9275.

Contacto: maria.pincay@unesum.edu.ec

Recibido: 25-04-2020

Aprobado: 23-06-2020

\begin{abstract}
Resumen
La hipertensión arterial (HTA) constituye un importante problema de salud pública, ya que es una enfermedad crónica y asintomática que requiere la adherencia de los pacientes al tratamiento de una manera permanente y sistemática para su control. El objetivo de este proyecto fue determinar el comportamiento de la adherencia terapéutica en pacientes con hipertensión arterial. Para ello se realizó un estudio descriptivo, sustentado en la revisión bibliográfica de investigaciones de tipo científico, relacionadas con el tema, seleccionándose para la investigación 39 publicados entre 2015 y 2020, entre los cuales están textos, páginas web, artículos de Medline Plus, Clinic Barcelona, Cuídate Plus, SciELO. La técnica utilizada fue la compilación bibliográfica. Los resultados que más destacan están los factores genéticos, socioeconómicos y su influencia negativa en la adherencia al tratamiento, debido al bajo nivel educativo y a la poca disponibilidad económica para cubrir los gastos que requiere el tratamiento; las características de la enfermedad crónica y frecuentemente asintomática dificulta la toma de conciencia; el ineficiente seguimiento a los pacientes y los insuficientes conocimientos sobre hipertensión arterial y su terapéutica, por parte de los profesionales, contribuyen a la falta de adherencia al tratamiento hipotensor,
\end{abstract}

Palabras claves; adherencia terapéutica, factores socioeconómicos, antihipertensivos, enfermedad crónica, asintomática.

Abstract
Hypertension (HT) constitutes an important public health problem, since it is a chronic and asymptomatic disease that requires adherence of patients to treatment in a permanent and systematic way for its control. The objective of this project was to determine the behavior of therapeutic adherence in patients with arterial hypertension. For this, a descriptive study was carried out, based on the bibliographic review of scientific research related to the subject, selecting for the research 39 published between 2015 and 2020, among which are texts, web pages, articles from Medline Plus, Clinic Barcelona, Take Care Plus, SciELO. The technique used was the bibliographic compilation. The results that stand out the most are the socioeconomic factors and their negative influence on adherence to treatment, due to the low educational level and the limited economic availability to cover the expenses required by the treatment; the characteristics of the chronic and frequently asymptomatic disease make awareness difficult; the inefficient follow-up of patients and insufficient knowledge about arterial hypertension and its therapy, by professionals, contribute to the lack of adherence to hypotensive treatment.

Keywords; therapeutic adherence, socioeconomic factors, antihypertensives, chronic, disease, asymptomatic.

\section{Introducción}

La Hipertensión Arterial (HTA), una de las enfermedades cardiovasculares catalogadas dentro de las enfermedades no transmisibles, con resultados epidemiológicos reconocidos, el mismo que ha tenido un aumento en su 
HIGÍA DE LA SALUD

prevalencia, asociado con un incremento en el envejecimiento de la población y a factores de riesgo, específicamente los relacionados el tabaquismo, la mala alimentación, la inactividad física, el consumo de alcohol y una falta de adherencia al tratamiento farmacológico.

Las guías clínicas vigentes sobre el tema hasta el año 2017, plantean que cuando el paciente manifiesta incrementos continuos de presión arterial (PA), donde la presión sistólica sostenida se encuentra por encima de $140 \mathrm{~mm} \mathrm{Hg}$ o la presión diastólica sostenida mayor de $90 \mathrm{~mm} \mathrm{Hg}$ (en al menos 3 visitas), se puede hablar de HTA . No obstante, en la guía aprobada en el año 2018, estos valores se reducen, considerando hipertensión arterial cuando estos valores son de $130 \mathrm{~mm} \mathrm{Hg} \mathrm{y} \mathrm{de} 80 \mathrm{~mm} \mathrm{Hg}$, para la presión arterial sistólica y diastólica, respectivamente (Diaz -Molina \& Polo-Vega, 2019)

Según la Organización Mundial de la Salud (OMS) y la Organización Pnamericana de la Salud(OPS) la hipertensión arterial afecta más del $30 \%$ de la población adulta mundial y es el principal factor de riesgo para padecer enfermedades cardiovasculares, especialmente enfermedad coronaria y enfermedad cerebrovascular, pero también para la enfermedad renal crónica, la insuficiencia cardíaca y la demencia. La carga de hipertensión recae de forma desproporcionada en países de ingresos bajos y medianos, que tienen dos tercios de los casos, en gran parte debido al incremento de los factores de riesgo en esas poblaciones en las últimas décadas

La hipertensión arterial (HTA) constituye un importante problema de salud pública en los países industrializados, se estima que alrededor de un $20 \%$ de la población adulta es hipertensa. Según la Organización Mundial de la Salud (OMS), uno de cada cinco adultos padece de tensión arterial elevada, un trastorno que ocasiona aproximadamente la mitad de todas las defunciones por accidente cerebrovascular o cardiopatía. Las complicaciones derivadas de la hipertensión son la causa de 9,4 millones de defunciones cada año en el mundo, problemática presente en varios países del mundo, y acentuada en Latinoamérica. (files.sld.cu)

En América Latina la prevalencia de hipertensión arterial es del $20 \%$. Entre los países que presentan cifras representativas de esta enfermedad son Cuba 44\%, Argentina 26 y $32 \%$, Brasil 22 y $32,7 \%$, Venezuela $15.9 \%$ y $39.2 \%$ y Ecuador con $28.7 \%$ en personas adultas. Se considera que una de cada dos personas que se diagnostica con hipertensión abandona el tratamiento antes de los seis meses, la mayor parte de la población lo hace porque refiere sentirse mejor, no presenta síntomas o manifiesta
Periodo. Enero - Junio 2020

Vol. 2, Nro. 1, Publicado 2020-06-30

que los fármacos le hacen daño (Carrillo Bayas, Determinantes de salud que influyen en la adherencia al tratamiento en pacientes con hipertensión, 2019)

Entre los factores de riesgo para enfermedad cardiovascular, la hipertensión es uno de los más importantes. Afecta a casi mil millones de personas y provoca el $8 \%$ de muertes en todo el mundo. El número total de adultos con hipertensión en todo el mundo en el 2000 fue de 972 millones; es probable que de acuerdo a estos datos aumente en un $60 \%$ a un total de 1560 millones en el 2025, La prevalencia en los estudios por la OMS fue de 10,4\% (Mujeres: 9,7\%; Hombres: $11,9 \%$ ) y de HTA conocida de 8,6\% (Mujeres: 9,7\%; Hombres: 6,3\%), representando una prevalencia global del $19 \%$ $(19,4 \%$ en mujeres y $18,2 \%$ en hombres) cifra que ha llevado a que la HTA se considere un problema de salud pública mundial tanto en países económicamente desarrollados así como en países en vías de desarrollo. (scielo, s.f)

En Ecuador el porcentaje de pacientes con hipertensión es un poco más elevado en hombres que mujeres. Desde el año 2015, la hipertensión arterial y otras patologías $\begin{array}{llll}\text { relacionadas superan } & \text { los } & \mathbf{7 . 5 0 0}\end{array}$ egresos hospitalarios al año. En el 2017 se reportaron 5.776 solo por hipertensión primaria, según el registro estadístico de Egresos Hospitalarios del Instituto Nacional de Estadísticas y Censos (INEC). (Silvano Bertozzi, 2019)

La hipertensión arterial es una enfermedad que ha ido aumentando a la par y como consecuencia del desarrollo económico mundial, la concentración de la población en las grandes ciudades, el envejecimiento y la globalización económica, estos estilos de vida son factores perjudiciales para la salud. (salud.gob.ec, 2018) $\mathrm{Su}$ prevalencia es de entre un $45 \%$ a un $30 \%$ dependiendo del desarrollo económico de cada país, siendo en general mayor en los países con menores ingresos. Además de ser la hipertensión una enfermedad en sí misma, se reconoce su carácter de factor de riesgo en el desarrollo de enfermedades cardiovasculares. (salud.gob.ec, 2018)

Según la OMS, la adherencia terapéutica se define como: el nivel del compromiso del paciente en cumplir las indicaciones de su médico en cuanto a la toma del medicamento, al cambio de su hábito alimenticio y estilo de vida sedentario (Carrillo Bayas, Determinantes de salud que influyen en la adherencia al tratamiento en pacientes con hipertensión, 2019). La adherencia es la estrategia que permite que el paciente mantenga y continúe el tratamiento para lograr cambios significativos en su salud, 
HIGÍA DE LA SALUD

mediante las recomendaciones de los profesionales de salud siendo así participe de su autocuidado.

La falta de adherencia a la terapia antihipertensiva contribuye directamente a que los pacientes coexistan con hipertensión, la misma que desencadena en eventos que ponen en riesgo de mayores morbilidades y mortalidad de estos pacientes. Así, la falta de adherencia al tratamiento se convierte en una de las principales causas de hipertensión no controlada en la población.

Por ello el comportamiento de adherencia a los tratamientos es uno de los indicadores más importantes a evaluar en las personas con factores de riesgo para la salud cardiovascular, ya que la no adherencia conduce a mantener altos índices de morbimortalidad, con repercusiones a nivel social, económico, individual y familiar. (scielo.isciii, 2018)

La adherencia al tratamiento médico indicado es fundamental para el control de la hipertensión arterial. Esta afirmación es reconocida por la mayoría de los profesionales de salud que atienden personas con esta enfermedad y en las informaciones sobre este tema.

Mediante Estudios realizados por el MSP en el Ecuador sobre la adherencia a los medicamentos en pacientes con hipertensión arterial se dice, que pacientes que están en el rango de edad de 41-60 años hubo un predominio de la adherencia parcial $(72,7 \%)$. Con respecto a los sujetos mayores de 60 años hubo un predominio de la adherencia parcial $(64,3 \%)$. Como dato relevante en este rango de edad un $(35,7 \%)$ de pacientes se adhirieron totalmente al tratamiento. Se ha demostrado que el médico dedica en general poco o nada de tiempo de laconsult a para evaluar la adherencia al tratamiento. Es re comendable la evaluación de la adherencia dur ante la consulta por medio de un cuestionari o validado. Para valorar adherencia se dispone de métodos directos e ind irectos. Los primeros si bien tienen la ventaja de tener un mayor grado de exactitud, prese ntan la limitación del costo o la disponibilid ad, como la búsqueda de fármacos en plasm a $\mathrm{u}$ orina. Los métodos indirectos presentan mayor accesibilidad y son aplicables en la consulta. (Sabio, 2018)El presente proyecto busca integrar las diferentes asignaturas tanto las relacionadas con el cuidado enfermero a nivel curativo como preventivo mediante Enfermería Médico quirúrgico II, enfermería en situación de riesgo y salud ocupacional, así como la gestión del cuidado enfermero a través de la administración de servicios de salud. Además de recibir la preparación necesaria mediante la pedagogía y didáctica para realizar actividades
Vol. 2, Nro. 1, Publicado 2020-06-30

de promoción de la salud y prevención de enfermedades entre ellas la de tipo sexual y reproductiva.

\section{Materiales y métodos}

Se realizó un estudio descriptivo, ya que detalla de forma clara todos los aspectos encontrados en el proceso de investigación para el análisis de los datos recolectados, así como para describir los diferentes resultados, sustentado en la revisión bibliográfica de investigaciones de tipo científico, relacionadas con el tema, en las cuales se consultaron diferentes fuentes, entre las cuales están las bases de datos de carácter internacional y nacional como: Medline Plus, Clinic Barcelona, Cuídate Plus, SciELO. Al realizar la búsqueda, se obtuvieron un total de 60 documentos de los cuales fueron seleccionados para la investigación 39 publicados entre 2015 y 2020.

Criterios de inclusión: Para la selección de las fuentes de información se tuvo en cuenta que las investigaciones no tuvieran más de cinco años de haber sido publicadas, y cuyo tema este enfocada en la temática Se incluyó Google Académico, como fuente de búsqueda de información. Criterios de Exclusión: Se excluyeron fuentes bibliográficas con más de 5 años de vigencia, trabajos, cuya base de datos no son de libre acceso, e información de fuentes no confiables. Los métodos utilizados fueron los empíricos (observacional) y teóricos (analítico y sintético), porque permiten el análisis de las investigaciones, mediante publicaciones indexadas en revistas científicas, seleccionando las que mejor información aportan para el desarrollo de la temática. La técnica utilizada es la compilación bibliográfica, lo que permitió realizar análisis y síntesis de documentos que muestran información confiable, entre los cuales están textos, páginas web, artículos etc.

\section{Resultados}

Esta revisión bibliográfica fue de gran ayuda para la estructura de construir conocimiento, recoger información sobre un determinado problema y procesarla de manera tal que agregue datos significativos para este estudio, y a la vez, fundamentar los procedimientos en la evidencia científica.

La ejecución del proyecto estuvo centrada en la adherencia al tratamiento de la hipertensión arterial, focalizándose en aquellos aspectos como la adherencia al tratamiento farmacológico y no farmacológico, descripción de los síntomas y complicaciones y la Identificación de las causas y complicaciones de la no adherencia. Cabe destacar que la ejecución del Proyecto, consideró los siguientes aspectos:

- Se desarrollaron todas las actividades sustantivas que fueron programadas, 
HIGIA DE LA SALUD obteniéndose la investigación establecida.

- Se ha contado con la participación de los docentes encargados de la revisión del proyecto y la validación y aprobación de la información.

- Se ha trabajado arduamente en el cumplimiento de todos los pasos para la
Periodo. Enero - Junio 2020

Vol. 2, Nro. 1, Publicado 2020-06-30

realización del proyecto en este periodo académico.

\begin{tabular}{|c|c|c|c|}
\hline Autor & Año & $\begin{array}{l}\text { Nombre de } \\
\text { investigación }\end{array}$ & Contenido de la publicación \\
\hline $\begin{array}{l}\text { Carmen } \\
\text { Ávila Lillo }\end{array}$ & 2015 & $\begin{array}{l}\text { Prevalencia de } \\
\text { personas con } \\
\text { hipertensión } \\
\text { arterial. }\end{array}$ & $\begin{array}{l}\text { El total de estudios consultados en relación a la edad el } \\
\text { número de hipertensos se incrementa con los años, así el } \\
\text { mayor porcentaje de hipertensos }(82 \%) \text { se corresponde con } \\
\text { el grupo de edad comprendido entre los } 66 \text { y } 85 \text { años y el } \\
\text { menor ( } 22 \%) \text { con el de edad comprendida entre los } 25 \text { y } 44 \\
\text { años. }\end{array}$ \\
\hline $\begin{array}{l}\text { Blanca } \\
\text { Estela } \\
\text { Fernández }\end{array}$ & 2015 & $\begin{array}{l}\text { Signos y } \\
\text { síntomas en } \\
\text { personas con } \\
\text { hipertensión } \\
\text { arterial }\end{array}$ & $\begin{array}{l}\text { La mayoría de personas son asintomáticas no presentan } \\
\text { signos de alerta, por lo que se dice que mata silenciosamente. } \\
\text { Suelen presentar síntomas como: cefaleas matutinas } \\
\text { hemorragias nasales, ritmo cardiaco irregular, la } \\
\text { hipertensión grave puede provocar fatiga, náuseas, vómitos, } \\
\text { confusión, ansiedad, dolor torácico y temblores musculares. }\end{array}$ \\
\hline $\begin{array}{l}\text { Carmen } \\
\text { Ávila Lillo }\end{array}$ & 2015 & $\begin{array}{l}\text { Adaptación al } \\
\text { nuevo estilo de } \\
\text { vida y control } \\
\text { médico } \\
\text { oportuno }\end{array}$ & $\begin{array}{l}\text { La incidencia de los distintos hábitos de vida: Tabaco, } \\
\text { obesidad, sedentarismo, alimentación, consumo de } \\
\text { sustancias excitantes, alcohol y actividad física, por lo que la } \\
\text { recomendación de mantener hábitos de vida saludables se ha } \\
\text { convertido en la piedra angular de los programas de } \\
\text { prevención de la HTA. Programas en los que el farmacéutico } \\
\text { puede jugar un papel relevante, tanto en la concienciación de } \\
\text { los pacientes respecto de las consecuencias de la } \\
\text { enfermedad, como en la detección de la HTA y en el } \\
\text { seguimiento del tratamiento. }\end{array}$ \\
\hline $\begin{array}{l}\text { Eugenia } \\
\text { Herrera } \\
\text { Guerra }\end{array}$ & 2017 & $\begin{array}{l}\text { Factores } \\
\text { riesgo y } \text { de } \\
\text { impacto } \\
\text { tratamiento de la } \\
\text { hipertensión } \\
\text { arterial }\end{array}$ & $\begin{array}{l}\text { Los factores socioeconómicos son determinantes poderosos } \\
\text { del estado de salud, que afectan los tratamientos médicos. } \\
\text { Existen factores socioeconómicos a los que se les atribuye } \\
\text { un efecto considerable sobre la adherencia al tratamiento. la } \\
\text { mayoría de las personas se encontró en riesgo de no } \\
\text { desarrollar comportamientos de adherencia al tratamiento } \\
\text { farmacológico y no farmacológico. }\end{array}$ \\
\hline $\begin{array}{l}\text { Dra. Liset } \\
\text { Jiménez } \\
\text { Fernández }\end{array}$ & 2017 & $\begin{array}{l}\text { Déficit de } \\
\text { conocimiento } \\
\text { por parte del } \\
\text { personal acerca } \\
\text { de la } \\
\text { enfermedad }\end{array}$ & $\begin{array}{l}\text { El ineficiente seguimiento a los pacientes y los insuficientes } \\
\text { conocimientos sobre hipertensión arterial y su terapéutica, } \\
\text { por parte de los profesionales, contribuyeron a la falta de } \\
\text { adherencia al tratamiento hipotensor, lo cual se relacionó } \\
\text { con un deficiente control de la enfermedad. }\end{array}$ \\
\hline $\begin{array}{l}\text { Panjabi S, } \\
\text { Lacey M, } \\
\text { Bancroft T }\end{array}$ & 2017 & $\begin{array}{l}\text { Causas y } \\
\text { complicaciones } \\
\text { de la adherencia } \\
\text { al tratamiento de } \\
\text { la hipertensión } \\
\text { arterial }\end{array}$ & $\begin{array}{l}\text { Si bien las causas de baja adherencia son variadas, se } \\
\text { destacan por su importancia aquellas relacionadas a las } \\
\text { características de la enfermedad crónica y frecuentemente } \\
\text { asintomática que dificulta la toma de conciencia. Otra causa } \\
\text { es vinculable al fármaco por su perfil de efectos adversos, } \\
\text { complejidad de dosificación (a mayor número de fármacos } \\
\text { peor adherencia) y mayor costo económico. }\end{array}$ \\
\hline Jennifer B & 2018 & $\begin{array}{l}\text { Promover un } \\
\text { estilo de vida en }\end{array}$ & $\begin{array}{l}\text { Cambiar el estilo de vida es muy importante en el control de } \\
\text { la presión arterial, llevar una dieta con menos sal saludable } \\
\text { para el corazón, realizar actividad física regular, mantener }\end{array}$ \\
\hline
\end{tabular}




\begin{tabular}{|c|c|c|c|}
\hline \multicolumn{4}{|c|}{ Vol. 2, Nro. 1, Publicado 2020-06-30 } \\
\hline & & $\begin{array}{l}\text { el paciente con } \\
\text { hipertensión }\end{array}$ & $\begin{array}{l}\text { un peso saludable o perder peso si tienes sobrepeso u } \\
\text { obesidad, y limitar la cantidad de alcohol que consumes. }\end{array}$ \\
\hline Wilfredo & 2018 & $\begin{array}{l}\text { Factores } \\
\text { mundiales ante } \\
\text { enfermedades } \\
\text { crónicas }\end{array}$ & $\begin{array}{l}\text { El fenómeno de la adherencia al tratamiento es un problema } \\
\text { mundial de alarmante magnitud, el cual está determinado por } \\
\text { la interacción de factores que influyen en el cuidado de } \\
\text { enfermedades crónicas, como la hipertensión arterial. }\end{array}$ \\
\hline $\begin{array}{l}\text { Oñate } \\
\text { Intriago } \\
\text { Angélica } \\
\text { Isabel }\end{array}$ & 2019 & $\begin{array}{lr}\text { Factores } & \text { que } \\
\text { originan } & \text { la } \\
\text { hipertensión } & \\
\text { arterial } & \end{array}$ & $\begin{array}{l}\text { En lo relacionado a factores de riesgo la deficiente o nula } \\
\text { actividad física, es uno de los principales factores que } \\
\text { producen la hipertensión arterial en conjunto a la mala } \\
\text { alimentación, la genética familiar y la falta de control } \\
\text { médico, los mismos que nos lleva al resultado que los } \\
\text { encuestados tienen el conocimiento neto de la procedencia } \\
\text { de su enfermedad y como se desarrolla en su salud normal. }\end{array}$ \\
\hline $\begin{array}{l}\text { Dr. C. Dany } \\
\text { Siverio } \\
\text { Mota }\end{array}$ & 2017 & $\begin{array}{l}\text { Aceptación y } \\
\text { colaboración del } \\
\text { paciente ante el } \\
\text { tratamiento } \\
\text { sugerido de la } \\
\text { OMS }\end{array}$ & $\begin{array}{l}\text { Hay una diferencia significativa entre adherencia y } \\
\text { cumplimiento. En este sentido, la OMS aclara que la } \\
\text { diferencia principal está dada por el hecho que la } \\
\text { adherencia requiere del consentimiento del paciente en } \\
\text { cuanto a las recomendaciones, mientras que el segundo } \\
\text { término implica que el propósito de recetar es conseguir que } \\
\text { el paciente siga las instrucciones del médico }\end{array}$ \\
\hline $\begin{array}{l}\text { Dr. Juan } \\
\text { Miguel } \\
\text { Chala } \\
\text { Tandrón }\end{array}$ & 2017 & $\begin{array}{l}\text { Cuidados } \\
\text { respectivos para } \\
\text { el paciente } \\
\text { según la OMS } \\
\text { con respecto a la } \\
\text { adherencia al } \\
\text { tratamiento }\end{array}$ & $\begin{array}{l}\text { La Organización Mundial de la Salud cuando plantea que } \\
\text { "las buenas relaciones entre los pacientes y sus prestadores } \\
\text { de asistencia sanitaria son por ende imperativas para la } \\
\text { buena adherencia, así como, la actitud y asistencia empática } \\
\text { y moralmente neutra, la pronta disponibilidad, la buena } \\
\text { calidad de la comunicación y la interacción son algunos de } \\
\text { los atributos importantes de los profesionales de asistencia } \\
\text { sanitaria que se ha demostrado son determinantes de la } \\
\text { adherencia de los pacientes. }\end{array}$ \\
\hline
\end{tabular}

\section{Conclusiones}

De acuerdo con la revisión bibliográfica realizada, más de la mitad de las personas con hipertensión arterial en el mundo no tienen un tratamiento efectivo. La falta de adherencia al tratamiento antihipertensivo, se manifiesta mayormente por una falta de voluntad entre los pacientes hipertensos refractarios, sin embargo la adherencia cobra importancia al comportarse como uno de los factores modificables que inciden directamente en los resultados del tratamiento. La adhesión al tratamiento es entendida como la colaboración y participación proactiva y voluntaria del paciente con su tratamiento para obtener unas mejores condiciones de salud y de vida, que van más allá del cumplimiento pasivo de las indicaciones del profesional de la salud. Entre los síntomas que se presentan en la hipertensión arterial tenemos visión borrosa, cefalea, dificultad para respirar o sangrado nasal, pero estos signos y síntomas no son específicos y, por lo general, no se presentan hasta que dicho trastorno alcanza una etapa grave o potencialmente fatal.

Una de las principales causas del fallo terapéutico es la poca adherencia al tratamiento farmacológico de pacientes hipertensos, las mismas que están relacionadas con las características de la enfermedad y de los pacientes, desconocimiento de la definición, secuelas y necesidades terapéuticas de la hipertensión, ausencia de síntomas, características del tratamiento, efectos adversos de la medicación, cronicidad del tratamiento y coste económico, así como la complejidad de las pautas terapéuticas. Todo esto conduce a mantener altos índices de morbimortalidad, con repercusiones a nivel social, económico, individual y familiar.

Colaboradores de la Investigación:

Jessenia Martínez Pilay, Gabriela Mejía Salazar, Michelle Ortega Chiquito, Abel Pincay Posligua, Jenniffer Ponce Choez. 
ISSN 2773-7705

\section{HIGIA DE LA SALUD}

Periodo. Enero - Junio 2020

Vol. 2, Nro. 1, Publicado 2020-06-30

\section{Referencias Bibliográficas}

1 Diaz -Molina M, Polo-Vega J. Adherencia a antihipertensivos en pacientes de la Aldea de Punuare, Departamento de Olancho, Honduras. Revista Salud Quintana Roo. 2019 Enero - junio ; 12(41).

2 files.sld.cu. files.sld.cu. [Online].; 2017 [cited 2020 6 28. Available from: https://files.sld.cu/bmn/files/2017/04/factografico-de-salud-abril-20171.pdf.

3 Carrillo Bayas G. Determinantes de salud que influyen en la adherencia al tratamiento en pacientes con hipertensión. Revista Mediciencias UTA. 2019; 3.

4 scielo. [Online].; $\quad$ s.f $\quad$ [cited 2020 julio 2. Available from: http://scielo.sld.cu/scielo.php?script=sci_arttext\&pid=S1029-30192019000400632.

5 Silvano Bertozzi. edicionmedica. [Online].; 2019 [cited 2020 julio 01. Available from: 5. https://www.edicionmedica.ec/secciones/profesionales/ecuador-tiene-una-prevalencia-de-9-3-porciento-de-hipertension-94139.

6 salud.gob.ec. salud.gob.ec. [Online].; 2018 [cited $2020 \quad 6 \quad 28$. Available from: https://www.salud.gob.ec/wp-content/uploads/2019/06/gpc_hta192019.pdf.

7 Carrillo Bayas G. Determinantes de salud que influyen en la adherencia al tratamiento en pacientes con hipertensión. Mediciencias UTA. 2019 junio ; 3.

8. scielo.isciii. scielo.isciii. [Online].; 2018 [cited 2020 6 28. Available from: http://scielo.isciii.es/pdf/enefro/v17n4/03_original2.pdf.

9 temas.sld. temas.sld. [Online].; 2017 [cited 2020 6 28. Available from: https://temas.sld.cu/hipertension/tag/oms.

10 revista medica. [Online].; s.f [cited 2020 julio 3. Available from: https://scholar.google.com.ec/scholar?q=Hipertensi\%C3\%B3n+arterial+adherencia+SCIELO+Ecuad or\&hl=es\&as_sdt=0\&as_vis=1\&oi=scholart\#d=gs_qabs\&u=\%23p\%3DU4o26HT310UJ.

11 Sabio R. Hipertensión arterial y adherencia al tratamiento: la brecha entre ensayos clínicos y realidad. Revista cubana de salud pública. 2018 Mar;(44). 